\title{
The Value of Two-Person Zero-Sum Repeated Games with Lack of Information on Both Sides ${ }^{1}$ )
}

\author{
By Jean-Francots Mertens and Shmuel Zamir
}

\begin{abstract}
We consider repeated two-person zero-sum games in which each player has only partial information about a chance move that takes place at the beginning of the game. Under some conditions on the information pattern it is proved that $\lim _{n \rightarrow \infty} v_{n}$ exists, $v_{n}$ being the value of the game with $n$ repetitions. Two functional equations are given for which $\lim v_{n}$ is the only simultaneous solutions. We also find the least upper bound for the error term $\mid \begin{gathered}n \rightarrow \infty \\ v_{n}-\lim _{n \rightarrow \infty} v_{n} \mid\end{gathered}$
\end{abstract}

\section{Introduction}

A game with incomplete information is a game in which one or more of the players do not know the complete description of the game; for instance, they do not know the other players' utility functions or their strategic possibilities. HARSANYI proposes a model for such games. The present study is concerned with what happens when such a game is played repeatedly. Though it is conceptually motivated by HARSANYI's theory, this paper is mathematically self-contained, and familiarity with HARSANYI's theory is not necessary to understand it.

HARSANYI shows that, granted some conditions and postulates, games of incomplete information are game-theoretically equivalent to certain games with complete information, which he calls "Bayesian Games". But when a game of incomplete information is played repeatedly, the unknown parameters of the game remain the same; they cannot change from stage to stage, be choosen again and again at each stage, as would be the case if the "Bayesian Game" with complete information was repeated. So, at each stage of the game, the players can learn something about the parameters originally unknown to them but known to the others, by watching which strategy the other players use.

Since quite many of these games are in fact repeated a large number of times, AUMANN and MASCHLER [1967] began to study the often-repeated games with incomplete information. In their attempt to find a suitable solution concept, they suggested two alternative approaches [AUMANN and MASCHLER, 1968]. The first is to consider the $n$-times repeated game $\Gamma_{n}$ and its value $v_{n}$ and then find out

\footnotetext{
$\left.{ }^{1}\right)$ This research was done during the visit of J. F. Mertens to the Institute of Mathematics of the Hebrew University of Jerusalem, March-April, 1970.
} 
$\lim _{n \rightarrow \infty}$ if it exists. The second approach is to treat directly the infinitely repeated $n \rightarrow \infty$ game $\Gamma_{\infty}$ and find its value $v_{\infty}$ when it exists.

In their first works on the subject, AUMANN and MASCHLER could not decide which approach to prefer since in the class of games they treated, namely 2-person 0 -sum with lack of information on one side, both $\lim v_{n}$ and $v_{\infty}$ exist and are equal.

For games with lack of information on both sides, the situation was already quite different. STEARNS has given a necessary and sufficient condition for the existence of $v_{\infty}$, and an example constructed by AUMANN, MASCHLER, and STEARNs showed that $v_{\infty}$ may not exist. But it was not known for that example whether $\lim v_{n}$ exists or not and still nothing could be said about the relation between the two approaches mentioned above.

An indication to such a relation was given by ZAMIR [1970] where an example was constructed in which $v_{\infty}$ does not exist while $\lim v_{n}$ exists. The example of ZAMIR [1970] does not belong to the class of games considered by STEARNS, namely it was not assumed that only choice of the pure strategies in each stage is told to the players. Nevertheless, that example led to the conjecture that in the STEARNS case the situation is similar. The present paper proves this conjecture. We prove that in a class of games which is larger than that treated in STEARNS, $\lim v_{n}$ exists and we give two functional equations for which $\lim v_{n}$ is the only simultaneous solution. An additional result is that the error term $\left|v_{n}-\lim v_{n}\right|$ is bounded by $(K / \sqrt{n})$ and this is the best bound since it is achieved.

In view of these results, $\lim v_{n}$ rather than $v_{\infty}$ seems to be the suitable concept of solution for a game of incomplete information repeated a large number of times.

\section{The Game}

In describing the class of games under consideration, let $\Gamma(p)$ denote the ordered five-tuple $\left\langle K, K^{\mathrm{I}}, K^{\mathrm{II}}, p, A\right\rangle$ where $K=\{1, \ldots, k\} . K^{\mathrm{I}}=\left\{K_{1}^{\mathrm{I}}, \ldots, K_{\mu}^{\mathrm{I}}\right\}$ and $K^{\mathrm{II}}=$ $\left\{K_{1}^{\mathrm{II}}, \ldots, K_{v}^{\mathrm{II}}\right\}$ are two partitions of the set $K$ into disjoint sets. $p=\left(p^{1}, \ldots, p^{k}\right)$ is a probability distribution on $K$ and $A=\left(A^{1}, \ldots, A^{k}\right)$ is a vector of $m \times l$ matrices of real numbers viewed as payoff matrices of 0-sum 2-person games. The elements of $A^{r}, r \in K$ will be denoted by $\left(a_{i j}^{r}\right), i \in\{1, \ldots, m\}=M, j \in\{1, \ldots, l\}=L$.

From $\Gamma(p)$ we derive a sequence of games $\left\{\Gamma_{n}(p)\right\}, n=1,2, \ldots \Gamma_{n}(p)$ is the $n$-times repeated game and is played as follows:

Stage- 0 . Chance chooses an element $r$ of $K$ according to the probability distribution $p$. Then player $I$ is informed of $\rho$ and player II is informed of $\eta$ where $r \in K_{\rho}^{\mathrm{I}} \cap K_{\eta}^{\mathrm{II}}$.

Stage-1. Player I chooses $i_{1}$ in $\{1, \ldots, m\}$ (Player I's first stage pure strategy) and player II chooses $j_{1}$ in $\{1, \ldots, l\}$ (Player II's first stage pure strategy). Both players are informed of $\left(i_{1}, j_{1}\right)$. Stage- 1 is repeated again and again. In the $h$-th stage, the players choose the pure strategies $i_{h}$ and $j_{h}$ respectively, then both are 
informed of $\left(i_{h}, j_{h}\right)$. After the $n$-th stage player I receives $\frac{1}{n_{h}} \sum_{h=1}^{n} a_{i_{h} j_{h}}^{r}$ from player II, where $r$ is the element chosen by chance in stage- 0 .

$a_{i n j_{n}}^{r}$ may be thought of as the payoff of player II to player I in the $h$-th stage of the game. However, it is important to notice that at any stage $h$ no player is informed directly of the payoff $a_{i_{h} j_{h}}^{r}$ at that stage. The division of $\sum_{h=1}^{n} a_{i_{h} j_{h}}^{r}$ by $n$ gives actually an average payoff per stage and this enables us to compare payoffs and values of $\Gamma_{n_{1}}(p)$ and $\Gamma_{n_{2}}(p)$ with $n_{1} \neq n_{2}$.

\section{Special Cases}

If $k=a b$ where $a$ and $b$ are positive integers, we may replace $K$ by $\{(\alpha, \beta) \mid \alpha=1, \ldots, a ; \beta=1, \ldots, b\}$. The probability distribution $p$ will then be written as a probability matrix $\left(r_{\alpha \beta}\right)$. Assume furthermore that $K^{\mathrm{I}}=\left\{K_{1}^{\mathrm{I}}, \ldots, K_{a}^{\mathrm{I}}\right\}$ and $K^{\mathrm{II}}=\left\{K_{1}^{\mathrm{II}}, \ldots, K_{b}^{\mathrm{II}}\right\}$ where $K_{\alpha}^{\mathrm{I}}=\{(\alpha, 1), \ldots,(\alpha, b)\} ; \alpha=1, \ldots, a$ and $K_{\beta}^{\mathrm{II}}=$ $\{(1, \beta), \ldots,(a, \beta)\} ; \beta=1, \ldots, b$. The class of games we get under these assumptions we shall call the "dependent product games". These games can be described as folinws: Player I can be one of $a$ different types $\mathrm{I}_{1}, \ldots, \mathrm{I}_{a}$ and player II can be one of $b$ different types $\mathrm{II}_{1}, \ldots, \mathrm{II}_{b}$. At stage- 0 chance chooses a pair of types $(\alpha, \beta)$ in accordance to the probability distribution matrix $\left(r_{\alpha \beta}\right)$. Player $I$ is informed of his type $\alpha$ and player II is informed of his type $\beta$ and the game then proceeds as described in the general case.

Notice that after stage- 0 player $I_{\alpha}$ (if chosen) will have the distribution $\left(r_{\alpha 1}, \ldots, r_{\alpha b}\right) / \sum_{\beta=1}^{b} r_{\alpha \beta}$ on the types of player II (and similarly for player II's distribution on the types of player I). This distribution is in general dependent of $\alpha$ i. e. on the type of player I. This explains the qualification "dependent" in the name of these games.

If, moreover, there exist two probability vectors $p=\left(p_{1}, \ldots, p_{a}\right)$ and $q=$ $\left(q_{1}, \ldots, q_{b}\right)$ such that $r_{\alpha \beta}=p_{a} q_{\beta}$ for $\alpha=1, \ldots, a ; \beta=1, \ldots, b$, we have then the "independent" case in which the probability distribution of each player is independent of his own type. These games we will call shortly "product games"; or, when ambiguity might arise, "independent product games". This is exactly the class of games considered by AUMANn and MASCHLER $[1967,1968]$, and by STEARns.

\section{The Main Theorem}

In order to state our main theorem let us introduce some more notations. Let $P$ denote the simplex:

$$
P=\left\{p=\left(p^{1}, \ldots, p^{k}\right) \mid p^{r} \geq 0 ; r=1, \ldots, k ; \sum_{r=1}^{k} p^{r}=1\right\} .
$$


For any two vectors $a=\left(a^{1}, \ldots, a^{k}\right)$ and $b=\left(b^{1}, \ldots, b^{k}\right)$ we denote by $a * b$ the vector $\left(a^{1} b^{1}, \ldots, a^{k} b^{k}\right)$ while $a \cdot b$ will denote as usual the scalar product $\sum_{r=1}^{k} a^{r} b^{r}$.

Now, given $\Gamma(p)=\left\langle K, K^{\mathrm{I}}, K^{\mathrm{II}}, p, A\right\rangle$ as described in $\S 1$, we define for any $p \in P$ two subsets of $P$ :

$$
\begin{gathered}
\Pi_{\mathrm{I}}(p)=\left\{\alpha * p \mid \alpha=\left(\alpha^{1}, \ldots, \alpha^{k}\right) ; \alpha^{r} \geq 0 ; \alpha \cdot p=1\right. \text { and: } \\
\left.i \in K_{\rho}^{\mathrm{II}} \text { and } j \in K_{\rho}^{\mathrm{II}} \text { for some } \rho \Rightarrow \alpha^{i}=\alpha^{j}\right\} . \\
\Pi_{\mathrm{II}}(p)=\left\{\begin{array}{c}
\left\{\beta * p \mid \beta=\left(\beta^{1}, \ldots, \beta^{k}\right) ; \beta^{r} \geq 0 ; \beta \cdot p=1\right. \text { and: } \\
\left.i \in K_{\eta}^{\mathrm{II}} \text { and } j \in K_{\eta}^{\mathrm{II}} \text { for some } \eta \Rightarrow \beta^{i}=\beta^{j}\right\} .
\end{array}\right.
\end{gathered}
$$

It is clear from the definition (2.1) that for any $p \in P$ both $\Pi_{\mathrm{I}}(p)$ and $\Pi_{\mathrm{II}}(p)$ are non-empty convex compact subsets of $P$. A function $f(p)$ defined on $P$ will be called concave with respect to I (shortly: w.r.t. I) if for any $p_{0} \in P, f(p)$ restricted to $\Pi_{\mathrm{I}}\left(p_{0}\right)$ is concave. Similarly, $f(p)$ will be called convex w.r.t. II if for any $p_{0} \in P$, $f(p)$ restricted to $\Pi_{\mathrm{II}}\left(p_{0}\right)$ is convex. Given any function $g(p)$ on $P$ we denote by $\underset{I}{\operatorname{Cav}} g(p)$ the minimal function $f(p)$ which satisfies:

i) $f(p) \geq g(p)$ for all $p \in P$

ii) $f(p)$ is concave w.r.t. I.

Similarly, we denote by $\underset{\text { II }}{\operatorname{ex}} g(p)$ the maximal function $f(p)$ which satisfies:

i) $f(p) \leq g(p)$ for all $p \in P$

ii) $f(p)$ is convex w.r.t. II.

Denote by $S$ and $T$ the sets of mixed strategies of the players in any game $A^{r}$ i.e. $S$ and $\boldsymbol{T}$ are the sets of probability distributions on $\{1, \ldots, m\}$ and on $\{1, \ldots, l\}$ respectively. In analogy to the STEARNS' case let $T_{\mathrm{I}}=\{1, \ldots, \mu\}$ and $T_{\mathrm{II}}=\{1, \ldots, \nu\}$ be called the sets of types of player I and of player II respectively. Denote by $K^{\mathbf{I}}$ the field generated by $K^{\mathbf{I}}$ and by $\boldsymbol{K}^{\mathbf{I I}}$ the field generated by $K^{\mathrm{II}}$.

Definition 1.

A one stage mixed strategy of player I is a $\boldsymbol{K}^{\mathbf{I}}$-measurable vector $\sigma=\left(\sigma^{1}, \ldots, \sigma^{k}\right)$ with $\sigma^{r} \in S(r=1, \ldots, k)$.

A one stage mixed strategy of player II is a $\boldsymbol{K}^{\mathbf{I I}}$-measurable vector $\tau=\left(\tau^{1}, \ldots, \tau^{k}\right)$ with $\tau^{r} \in T(r=1, \ldots, k)$. The sets of one stage mixed strategies of the players will be denoted by $S^{\mathbf{I}}$ and $T^{\mathbf{I I}}$.

\section{Definition 2.}

A strategy $\sigma=\left(\sigma^{1}, \ldots, \sigma^{k}\right)$ with $\sigma^{r} \in S(r=1, \ldots, k)$ is called non-separating (shortly NS) if $\forall i, j, \sigma^{i}=\sigma^{j}$. Similarly for $\tau=\left(\tau^{1}, \ldots, \tau^{k}\right)$ with $\tau^{r} \in T$. The sets of NS strategies will be denoted by $S^{*}$ and $T^{*}$.

\section{Definition 3.}

For any $p \in P$ we denote by $\Delta(p)$ the game $\Gamma_{1}(p)$ in which the players' sets of strategies are $S^{*}$ and $T^{*}$ (rather than $S^{\mathbf{I}}$ and $T^{\mathbf{I I}}$ ). Let $u(p)$ be the value of $\Delta(p)$ 
$\left(u(p)\right.$ is well defined and is continuous on $P$ ). Finally, let $v_{n}(p)$ be the value of $\Gamma_{n}(p)$ which surely exists and is continuous since $\Gamma_{n}(p)$ is a finite 0 -sum 2-person game. Now we are ready to state:

Theorem 2.1. (The main theorem).

$\lim v_{n}(p)$ exists and it is the only simultaneous solution of the functional $\lim _{n \rightarrow \infty}$ equations:
i) $v(p)=\underset{\text { II }}{\operatorname{ex}} \max \{u(p), v(p)\}$
ii) $v(p)=\underset{\mathrm{I}}{\mathrm{Cav}} \min \{u(p), v(p)\}$

\section{A Basic Theorem}

Before proving theorem 2.1, let us first prove a basic theorem that establishes the possibility of using a posteriori probabilities as state variables in $\Gamma_{n}(p)$. Essentially, the theorem introduces a MARKOV property.

Before the $m$-th stage of the game both players remember the pure strategies which have been chosen by both of them in previous $(m-1)$ stages: $\lambda_{m}=$ $\left[\left(i_{1}, j_{1}\right) ; \ldots ;\left(i_{m-1}, j_{m-1}\right)\right] . \lambda_{m}$ will be called the $m$-stage history. We denote by $\Lambda_{m}$ the set of all $m$-stage histories. In addition to $\lambda_{m}$ each player remembers his type as told to him in stage- 0 . Therefore, the information of each player before the $m$-th stage is an element of $T_{\mathrm{I}} \times \Lambda_{m}$ or $T_{\mathrm{II}} \times \Lambda_{m}$ respectively. Since $\Gamma_{n}(p)$ is a game with perfect recall we can without any loss of generality consider only behavioral strategies. A behavioral strategy of player $\mathrm{I}$ is an $n$-tuple $\left(f_{1}, \ldots, f_{n}\right)$ where $f_{m}$ $(m=1, \ldots, n)$ are functions $f_{m}: T_{\mathbf{I}} \times \Lambda_{m} \rightarrow S$ or equivalently $f_{m}: A_{m} \rightarrow S^{\mathbf{I}}$. Similarly, a behavioral strategy of player II is $\left(g_{1}, \ldots, g_{n}\right)$ where $g_{m}: A_{m} \rightarrow T^{\text {II }}$ $(m=1, \ldots, n)$.

Given two strategies $f=\left(f_{1}, \ldots, f_{n}\right)$ of player I and $g=\left(g_{1}, \ldots, g_{n}\right)$ of player II which are being played and given an $m$-stage history $\lambda_{m}$ we denote by $p_{m}$ the conditional distribution on $K$ conditionally to $\lambda_{m}$, where the probability space is $K \times A_{m}$, with probability derived from $p, f$, and $g$.

The probability distributions $\left\{p_{m}\right\}$ are actually the "state variables" of the game in the sense of theorem 3.1 .

\section{Lemma.}

The game $\Gamma_{n}(p)$ has the same value as the following game: First $\Gamma_{m-1}(p)$ $(1 \leq m \leq n)$ is played, the players tell their (behavioral) strategies to the referee, then the a posteriori probability $p_{m}$ is told to both players by the referee and they start playing $\Gamma_{n-m+1}\left(p_{m}\right)$. The payoff of the whole game is $\left((m-1) H_{1}+\right.$ $\left.(n-m+1) H_{2}\right) / n$ where $H_{1}$ is the payoff in $\Gamma_{m-1}$ and $H_{2}$ is the payoff in $\Gamma_{n-m+1}$.

Proof.

The proof of the above theorem will be done in several steps. 


\section{Proposition $A$.}

The game $\Gamma_{n}(p)$ has the same value as $\Gamma_{n}^{\prime}(p)$ played as follows: First $\Gamma_{m-1}(p)$ is played, say with the strategies $\sigma_{m-1}$ and $\tau_{m-1}$. The payoff is multiplied by $(m-1) / n$. Then a new lottery is performed: First nature chooses an element $r$ of $K$ with the probability distribution $p$, tells the players their types and then chooses a history $\lambda_{m} \in \Lambda_{m}$ according to the conditional probability distribution given $r$ determined by $p, \sigma_{m-1}$ and $\tau_{m-1} . \lambda_{m}$ is told to both players and then they play another $(n-m+1)$ stages of the game and the payoffs are determined by the subgame chosen in the last lottery, multiplied by $1 / n$, and added to the payoff of the first part.

\section{Remark.}

It is not assumed that at stage $m$ both players know $\sigma_{m-1}$ and $\tau_{m-1}$. Only nature knows them.

\section{Proof of Proposition $A$.}

There is a one to one mapping of the sets of strategies of $\Gamma_{n}(p)$ into those of $\Gamma_{n}^{\prime}(p)$, namely: Let $f=\left(f_{1}, \ldots, f_{n}\right)$ be a strategy of player $\mathrm{I}$ in $\Gamma_{n}(p)$ then the corresponding strategy in $\Gamma_{n}^{\prime}(p)$ is $\left(f_{1}, \ldots, f_{m-1}\right)$ for the first part and $\left(f_{m}, \ldots, f_{n}\right)$ for the second. Since $f_{l}$ is a function from $T_{1} \times \Delta_{l}$ to $S$ it is a right strategy also in $\Gamma_{n}^{\prime}(p)$.

Now, the second lottery of nature is constructed so that the expected payoff for $f$ and $g$ in each stage of $\Gamma_{n}(p)$ is equal to the expected payoff in the same stage in $\Gamma_{n}^{\prime}(p)$ for the corresponding strategies $f^{\prime}$ and $g^{\prime}$.

In order to prove proposition $A$, it is now sufficient to show that if some strategy $f$ of player I in $\Gamma_{n}(p)$ guarantees some payoff $M_{f}$ in $\Gamma_{n}(p)$, then $f^{\prime}$ guarantees also $M_{f}$ in $\Gamma_{n}^{\prime}(p)$. Indeed, the dual result for player II will then follow, and an application of the minimax theorem in $\Gamma_{n}(p)$ will complete the proof.

Since player II can now be assumed to know $f^{\prime}$, he knows the conditional distribution as a function of $r$ with which nature chooses $\lambda_{m} \in A_{m}$ at stage $m$. But then all the information (type and history) he got in the first $(m-1)$ stages, becomes completely irrelevant at stage $m$, since the second part of the game is determined by the new choice of nature, the probability distribution of which he knows independently of this information. So, player II can do no better against $f^{\prime}$ than with some strategy $g^{\prime}$. But then the payoff is the payoff resulting from $f$ and $g$, which is by assumption $\geq M_{f}$.

So Proposition A is proved.

\section{Proposition $B$.}

$v_{n}(p)$ - the value of $\Gamma_{n}(p)$ - is also the value of the following game $\Gamma_{n}^{\prime \prime}(p)$ : First $\Gamma_{m-1}(p)$ is played, say with mixed strategies $\sigma_{m-1}$ and $\tau_{m-1}$, the payoff is multiplied by $(m-1) / n$. Then a new lottery is performed: First, nature chooses a history $\lambda_{m} \in \Lambda_{m}$ according to the probability induced on $\Lambda_{m}$ by $p, \sigma_{m-1}$, and $\tau_{m-1}$. This history is told to both players, then nature chooses $r \in K$ according to the 
conditional probability on $K$ given $\lambda_{m}$ as determined by $p, \sigma_{m-1}$, and $\tau_{m-1}$. Both players are told their types and then they play another $(n-m+1)$ stages of the game where the payoffs are determined by the game choosen in the last lottery of nature, multiplied by $1 / n$, and added to the payoff of the first part.

Proof of Proposition $B$.

The only difference between $\Gamma_{n}^{\prime}(p)$ and $\Gamma_{n}^{\prime \prime}(p)$ is in the order in which nature chooses the game and the history and tells it to the players. This order is obviously irrelevant since the players have nothing to do in between. (The joint probability of game and history is the same in both cases).

Proof of the Lemma.

Propositions A and B show that the minimax theorem is valid in $\Gamma_{n}^{\prime \prime}(p)$. Therefore, we can assume from now on that in $\Gamma_{n}^{\prime \prime}(p)$, before stage $m$, both players know $p_{m}$.

In $\Gamma_{n}^{\prime \prime}(p)$, both players can then obviously forget the history $\lambda_{m}$ told by nature before the $m$-th stage, remembering only the conditional probability $p_{m}$ on $K$ given this history. So, in $\Gamma_{n}^{\prime \prime}(p)$ we can assume that nature only chooses a $p_{m}$ according to the distribution induced by $p, \sigma_{m-1}$, and $\tau_{m-1}$ on $\left\{p_{m}\right\}$, tells the $p_{m}$ to both players and then chooses a game according to $p_{m}$. But this choice of $p_{m}$ can surely be done in the following way: Nature - or the referee - watches the moves of both players in the first $m-1$ stages, and announces $p_{m}$ as computed from them knowing $p, \sigma_{m-1}$, and $\tau_{m-1}$. This completes the proof of the lemma.

Theorem 3.1.

$\Gamma_{n}(p)$ has the same value as the game in which at each stage $m$ the a posteriori probability $p_{m}$ is told to both players, and the payoff corresponding to that stage is determined by a new lottery on $K$ according to $p_{m}$.

Proof.

The theorem is proved by applying the lemma again and again.

\section{Proof of Theorem 2.1.}

Let $p_{m}$ be the a posteriori probability distribution on $K$ before the $m$-th stage and let $\sigma=\left(\sigma^{1}, \ldots, \sigma^{k}\right)$ and $\tau=\left(\tau^{1}, \ldots, \tau^{k}\right)$ be the two mixed strategies played by the players at stage- $m$. The expected payoff at that stage will be $H_{m}(\sigma, \tau)=$ $\sum_{r=1}^{k} p_{m}^{r} \sigma^{r} A^{r} \tilde{\tau}^{r}(\tilde{\tau}$ is the transposition of $\tau)$. The a posteriori probability after stage- $m$ will be $p_{m+1}$ from which each player will calculate conditional a posteriori probability given his type.

\section{Lemma 1.}

i) $\sigma$ is NS $\Rightarrow p_{m+1} \in \Pi_{\mathbf{I I}}\left(p_{m}\right) \Leftrightarrow$ A posteriori probability of II is unchanged after the $m$-th stage. 
ii) $\tau$ is $\mathrm{NS} \Rightarrow p_{m+1} \in \Pi_{\mathbf{I}}\left(p_{m}\right) \Leftrightarrow$ A posteriori probability of $\mathbf{I}$ is unchanged after the $m$-th stage.

This proposition is an immediate consequence of the definitions.

\section{Lemma 2.}

Let $p=\sum_{h=1}^{\mu} \lambda_{h} p_{h}$ where $p_{h} \in \Pi_{\mathrm{I}}(p), \lambda_{h} \geq 0,(h=1, \ldots, \mu)$, and $\sum_{h} \lambda_{h}=1$, then player I has a type dependent lottery on the set $\{1, \ldots, \mu\}$ such that:

i) The total probability of $h(h=1, \ldots, \mu)$ is $\lambda_{h}$.

ii) The conditional probability distribution on $K$ given the outcome $h$ is $p_{h}$ $(h=1, \ldots, \mu)$.

\section{Proof.}

Let $p_{h}=\alpha_{h} * p(h=1, \ldots, \mu)$. Since the components of $\alpha_{h}$ corresponding to the same set $K_{\rho}^{\mathbf{I}}$ are the same; we write $\alpha_{h}$ shortly as $\left(\alpha_{h}^{1}, \alpha_{h}^{2}, \ldots, \alpha_{h}^{\mu}\right)$. The required lottery on $\{1, \ldots, \mu\}$ is $\gamma_{\rho}=\left(\gamma_{\rho}^{1}, \gamma_{\rho}^{2}, \ldots, \gamma_{\rho}^{\mu}\right)$ for type $\rho(\rho=1, \ldots, \mu)$ where: $\gamma_{\rho}^{h}=\lambda_{h} \alpha_{h}^{\rho}$; $h=1, \ldots, \mu, \rho=1, \ldots, \mu$. Clearly, $\gamma_{\rho}^{h} \geq 0$ and also: $p=\sum_{h=1}^{\mu} \lambda_{h} p_{h}=\sum_{h=1}^{\mu} \lambda_{h}\left(\alpha_{h} * p\right)=$ $\left(\sum_{h=1}^{\mu} \lambda_{h} \alpha_{h}\right) * p$, hence, $(1, \ldots, 1)=\sum_{h=1}^{\mu} \lambda_{h} \alpha_{h}=\left(\sum_{h=1}^{\mu} \gamma_{1}^{h}, \ldots, \sum_{h=1}^{\mu} \gamma_{\mu}^{h}\right)$ which shows that $\gamma_{\rho}(\rho=1, \ldots, \mu)$ are probability distributions. To show (i) set $p^{(\rho)}=\sum_{i \in K_{\rho}^{\mathbf{j}}} p^{i}$, then the total probability of the outcome $h$ is $\sum_{\rho=1}^{\mu} p^{(\rho)} \gamma_{\rho}^{h}=\lambda_{h} \sum_{\rho=1}^{\mu} p^{(\rho)} \alpha_{h}^{\rho}=\lambda_{h}\left(p \cdot \alpha_{h}\right)=\lambda_{h}$ as required. To prove (ii) note that since the lottery depends only on the type it is clear that the distributions given the types are unchanged which means that the resulting distributions on $K$ are in $\Pi_{\mathrm{I}}(p)$. It suffices to prove therefore, that Prob $\left\{r \in K_{\rho}^{1} \mid h\right\}=p_{h}^{(\rho)}$. In fact:

$$
\operatorname{Prob}\left\{r \in K_{\rho}^{\mathrm{I}} \mid h\right\}=\frac{p^{(\rho)} \gamma_{\rho}^{h}}{\sum_{\rho} p^{(\rho)} \gamma_{\rho}^{h}}=\frac{p^{(\rho)} \lambda_{h} \alpha_{h}^{\rho}}{\lambda_{h}}=p^{(\rho)} \alpha_{h}^{\rho}=p_{h}^{(\rho)} .
$$

So the lemma is proved.

In what follows, we work on $\Omega=K \times L \times M$, where $L=\{1, \ldots, l\}$ and $M=\{1, \ldots, m\}$ stand for the pure strategy choices of both players at stage $m$.

Expectation with respect to the variables $\nu, \rho \ldots$ will be denoted by $E_{v, \rho} \ldots(\cdot)$. Let $\boldsymbol{M}$ and $\boldsymbol{L}$ be the $\sigma$-fields on $\Omega$ generated by the factors $M$ und $L$ respectively. The strategies $\sigma=\left(\sigma^{1}, \ldots, \sigma^{k}\right)$ and $\tau=\left(\tau^{1}, \ldots, \tau^{k}\right)$ played at stage- $m$ together with $p_{m}$ define a probability measure on $\Omega$. We shall be mainly interested in the expectation: $E_{i, j}\left(\left|p_{m}(r \mid \boldsymbol{M} \times \boldsymbol{L})-p_{m}(r)\right|\right)$ where $i$ and $j$ are the variables of $\boldsymbol{M}$ and $L$ respectively. This is the same as $E\left(\left|p_{m+1}(r)-p_{m}(r)\right|\right)$.

\section{Lemma 3.}

Let $\sigma=(\sigma, \ldots, \sigma)=\sigma\left(p_{m}\right)=\left\{s_{i}\left(p_{m}\right)\right\}, i \in M$ be an optimal strategy of player I in $\Delta\left(p_{m}\right)$. For any $\tau=\left(\tau^{1}, \ldots, \tau^{k}\right)$ we have 


$$
H_{m}(\sigma, \tau) \geq u\left(p_{m}\right)-\sum_{r \in K} c_{r} E\left(\left|p_{m+1}(r)-p_{m}(r)\right|\right)
$$

where $c_{r}=\max _{j, p}\left|a_{j}^{r}(p)\right|, a_{j}^{r}(p)=\sum_{i} s_{i}(p) a_{i j}^{r}$.

Proof.

Let $\bar{\tau}$ be defined by:

$$
\bar{\tau}=\bar{\tau}^{r}=\sum_{\rho \in K} p_{m}^{\rho} \tau^{\rho} ; \quad r=1, \ldots, k .
$$

By its definition $\bar{\tau}$ in NS and hence, a strategy of player II in $\Delta\left(p_{m}\right)$.

Now, if $\sigma=\left\{s_{i}\right\}, \tau^{r}=\left\{t_{j}^{r}\right\}, \bar{\tau}=\left\{\bar{t}_{i}\right\}, i \in M, j \in L, r \in K$, we have

$$
\begin{gathered}
p_{m+1}(r \mid i, j)=p_{m}^{r} s_{i} t_{j}^{r} / \sum_{\rho \in K} p_{m}^{\rho} s_{i} t_{j}^{\rho}=p_{m}^{r} t_{j}^{r} / \sum_{\rho \in K} p_{m}^{\rho} t_{j}^{\rho}=p_{m}^{r} \frac{t_{j}^{r}}{\bar{t}_{j}} \\
\left|p_{m+1}(r \mid i, j)-p_{m}(r)\right|=\left|t_{j}^{r} / \bar{t}_{j}-1\right| p_{m}(r) .
\end{gathered}
$$

So: $E\left(\left|p_{m+1}(r)-p_{m}(r)\right|\right)=\sum_{j=1}^{l}\left|t_{j}^{r} / \bar{t}_{j}-1\right| p_{m}(r) \operatorname{prob}(j)$.

But $\operatorname{prob}(j)=\bar{t}_{j}$

so we get:

$$
E\left(\left|p_{m+1}(r)-p_{m}(r)\right|\right)=p_{m}^{r} \sum_{j}\left|t_{j}^{r}-\bar{t}_{j}\right|
$$

Now, the expected payoff $H_{m}(\sigma, \tau)$ is:

$$
H_{m}(\sigma, \tau)=\sum_{r \in K} p_{m}^{r} \sigma A^{r} \tilde{\tau}^{r}=\sum_{r} p_{m}^{r} \sigma A^{r} \tilde{\tilde{\tau}}+\sum_{r} p_{m}^{r} \sigma A^{r}\left(\tilde{\tau}^{r}-\tilde{\tilde{\tau}}\right) .
$$

$\bar{\tau}$ is NS and $\sigma$ is optimal in $\Delta\left(p_{m}\right)$; therefore, the first term is at least $u\left(p_{m}\right)$. Now:

$$
\left|\sum_{r} p_{m}^{r} \sigma A^{r}\left(\tilde{\tau}^{r}-\tilde{\tau}\right)\right| \leq \sum_{r} p_{m}^{r} c_{r} \sum_{j}\left|t_{j}^{r}-\bar{t}_{j}\right|=\sum_{r} c_{r} E\left(\left|p_{m+1}(r)-p_{m}(r)\right|\right) .
$$

Inserted in (4.5), this completes the proof of lemma 3.

\section{Lemma 4.}

$$
\frac{1}{n} \sum_{m=1}^{n} \sum_{r \in K} c_{r} E\left(\left|p_{m+1}(r)-p_{m}(r)\right|\right) \leq \frac{1}{\sqrt{n}} \sum_{r \in K} c_{r} \sqrt{p^{r}\left(1-p^{r}\right)} \leq \frac{A \sqrt{k-1}}{\sqrt{n}}
$$

where $A=\max _{i, j, r}\left|a_{i, j}^{r}\right|$.

Proof.

Obviously, the sequence $p_{m}(r)$ is a martingale; therefore,

$$
E\left(\sum_{m=1}^{n}\left(p_{m+1}(r)-p_{m}(r)\right)^{2}\right)=E\left(p_{n+1}(r)-p_{1}(r)\right)^{2} \leq p^{r}\left(1-p^{r}\right)
$$

and by HöLDER's inequality:

$$
E\left(\sum_{m=1}^{n} \frac{1}{n}\left|p_{m+1}(r)-p_{m}(r)\right|\right) \leq \sqrt{E\left(\sum_{m=1}^{n} \frac{1}{n}\left(p_{m+1}(r)-p_{m}(r)\right)^{2}\right)}
$$

and hence, the first inequality. The second inequality is a simple majorization. 
Let

$$
\begin{aligned}
& \underline{v}(p)=\liminf v_{n}(p) \\
& \bar{v}(p)=\lim \sup v_{n}(p)
\end{aligned}
$$

\section{Lemma 5.}

The $v_{n}(p), n=1,2, \ldots$ are continuous functions on $P$ which have uniformly the LIPSCHITZ property, i.e., the LIPSCHITz constant can be assumed to be independent of $n$. So $\bar{v}(p)$ and $\underline{v}(p)$ also have this LIPSCHITZ property.

Proof.

Such a common LIPSCHITZ constant is $2 \mathrm{~A}$.

\section{Lemma 6.}

Let $f(p)$ be any function on $P$ such that $f(p) \leq \operatorname{Cav}_{i} \min \{u(p), f(p)\}$ then for any $p_{0} \in P$ and for any $\varepsilon>0$ there are $p_{i} \in \Pi_{\mathrm{I}}\left(p_{0}\right)(1 \leq i \leq \mu)$ and $\lambda_{i} \geq 0, \sum \lambda_{i}=1$ such that $\sum_{I}^{\mu} \lambda_{i} p_{i}=p_{0}$ and $f\left(p_{0}\right)-\varepsilon \leq \sum_{1}^{\mu} \lambda_{i} \min \left\{u\left(p_{i}\right), f\left(p_{i}\right)\right\}$.

Proof.

The proof is straightforward by CARATHEODORY's theorem, since $\Pi_{I}\left(p_{0}\right)$ is $(\mu-1)$ dimensional. (The involvement of $\varepsilon$ is necessary since $f(p)$ is not assumed to be continuous).

Proposition 4.1.

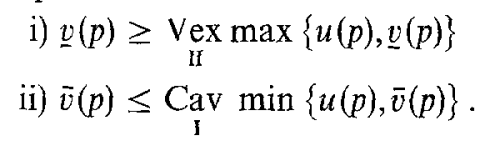

\section{Proof.}

We shall prove only the first inequality; the second will follow by duality considerations. To prove (i), we shall show that for any $\varepsilon>0$ we can find $N$ s.t. for any $n>N$ player I has a strategy in $\Gamma_{n}(p)$ that guarantees him $\underset{\text { II }}{\operatorname{ex}} \max \{u(p)$, $\underline{v}(p)\}-\varepsilon$. This will imply (i) since $\lim \inf v_{n}(p)$ is the highest payoff that player I can guarantee as $n \rightarrow \infty$ (up to an $\varepsilon$ ).

Define $\delta(p, n)=\left(\underline{v}(p)-v_{n}(p)\right)^{+}$.

Given $\varepsilon>0$, let $N_{1}(\varepsilon)$ be such that $n \geq N_{1}(\varepsilon) \Rightarrow \max _{p \in P} \delta(p, n)<\frac{\varepsilon}{3}$. (Since the $\delta(p, n)$ are uniformly LIPSCHITzian, by lemma 5 , they converge uniformly to zero.) Define $N=N(\varepsilon)=\max \left\{k\left(\frac{3 A}{\varepsilon}\right)^{2}, N_{1}(\varepsilon) \frac{6 A}{\varepsilon}\right\}$.

Take now a game $\Gamma_{n}(p)$ for $n \geq N$, and consider its equivalent game described in theorem 3.1. Let $f=\left(f_{1}, \ldots, f_{n}\right)$ be the following strategy of player $I:$ At stage- $m$ play optimally in $\Delta\left(p_{m}\right)$ as long as $u\left(p_{m}\right) \geq \underline{v}\left(p_{m}\right)$, and start playing an optimal strategy in the remaining game as soon as $u\left(p_{m}\right)<\underline{v}\left(p_{m}\right)$. 
Let $m_{0}=\min \left\{m \mid u\left(p_{m}\right)<\underline{v}\left(p_{m}\right)\right\}$. By lemma 3 , the expected payoff resulting from the above strategy is at least:

$\frac{1}{n} E\left\{\sum_{1}^{m_{0}} u\left(p_{m}\right)-\sum_{1}^{m_{0}} \sum_{r \in K} c_{r} E\left(\left|p_{i+1}(r)-p_{i}(r)\right|\right)+\left(n-m_{0}\right)\left(\underline{v}\left(p_{m_{0}}\right)-. \delta\left(p_{m_{0}}, n-m_{0}\right)\right\}\right.$.

Since up to stage- $m_{0}$ player I is playing NS, we have by lemma $1 p_{m} \in \Pi_{\mathrm{II}}(p)$ for $m=1, \ldots, m_{0}$, hence,

$$
\frac{1}{n} E\left(\sum_{1}^{m_{0}} u\left(p_{0}\right)+\left(n-m_{0}\right) \underline{v}\left(p_{m_{0}}\right)\right) \geq \operatorname{Vex}_{\mathrm{II}} \max \{u(p), \underline{v}(p)\}
$$
by lemma 4 , the second term in (4.8) is at most $A \frac{\sqrt{k}}{\sqrt{n}} \leq \frac{\varepsilon}{3}$. As for the last term
in $(4.8)$ we have:

$$
\begin{aligned}
E\left(\frac{n-m_{0}}{n} \delta\left(p_{m_{0}}, n-m_{0}\right)\right) & \leq \sum_{i=1}^{n-N_{1}(\varepsilon)}\left[\max _{p} \delta(p, n-i)\right] p\left(m_{0}=i\right) \\
& +\frac{N_{1}(\varepsilon)}{n} \sum_{n-N_{1}+1}^{n}\left[\max _{p} \delta(p, n-i)\right] p\left(m_{0}=i\right)
\end{aligned}
$$

(by definition of $N_{1}(\varepsilon)$ :)

$$
\leq \frac{\varepsilon}{3}+\frac{N_{1}(\varepsilon) \cdot 2 A}{n} \leq \frac{\varepsilon}{3}+\frac{\varepsilon}{3}
$$

We conclude that the above described strategy guarantees player I the quantity (4.8) which is $\geq \operatorname{Vex} \max \{u(p), \underline{v}(p)\}-\varepsilon$. This completes the proof of propotion 4.1.

\section{Proposition $4.2^{1}$ ).}

$\underline{v}(p)$ and $v_{n}(p) ; n=1,2, \ldots$ are concave w.r.t. $1 ; \bar{v}(p)$ and $v_{n}(p) ; n=1,2, \ldots$ are convex w.r.t. II.

\section{Proof.}

Since the maximum of two convex functions is convex, and the minimum of two concave functions is concave, it is sufficient to prove the proposition for $v_{n}(p)$. By duality considerations it is sufficient to prove the concavity only.

Assume on the contrary that there exist $p_{0} \in P, p_{i} \in \Pi_{\mathrm{I}}\left(p_{0}\right)(1 \leq i \leq \mu)$, and $\lambda_{i} \geq 0, \sum_{1}^{\mu} \lambda_{i}=1$ such that $\sum_{1}^{\mu} \lambda_{i} p_{i}=p_{0}$ and $\sum_{1}^{\mu} \lambda_{i} v_{n}\left(p_{i}\right)>v_{n}\left(p_{0}\right)$. Let us show that player.I can guarantee himself the quantity $\sum_{1}^{\mu} \lambda_{i} v_{n}\left(p_{i}\right)$ in $\Gamma_{n}\left(p_{0}\right)$. The way to do this is to make the lottery described in lemma 2 , after the initial move of chance,

\footnotetext{
1) This proposition for games with lack of information on one side is proved by AUMANN and MASCHLER [1968], p. 74].
} 
but before the first stage of the game. Then, if the outcome of the lottery is $i$ $(1 \leq i \leq \mu)$, he should play optimally in $\Gamma_{n}\left(p_{i}\right)$. Now, assume a fortiori that player II knew the outcome of this lottery. Then after the lottery, the situation is exactly as if both players started playing $\Gamma_{n}\left(p_{i}\right)$, so player $I$ can guarantee himself $v_{n}\left(p_{i}\right)$, and thus in average $\sum_{1}^{\mu} \lambda_{i} v_{n}\left(p_{i}\right)$.

\section{Proposition 4.3.}

Let $f$ be any function on $P$ such that $f(p) \leq \operatorname{Vex}$ Cav min $\{u(p), f(p)\}$. Define $\delta(p, n)=\left(f(p)-v_{n}(p)\right)^{+}$then $\delta(p, n) \leq \frac{1}{\sqrt{n}} \sum_{r \in K} c_{r} \sqrt{p^{r}\left(1-p^{r}\right)}$ in particular we
have then $f(p) \leq \underline{v}$

\section{Proof.}

Obviously, this set of functions $f$ has a largest element, for which the equality $f(p)=\underset{\text { II }}{\operatorname{Vex}} \operatorname{Cav} \min \{u(p), f(p)\}$ must hold. We can and do assume that we are dealing with this $f$. Let $\left.\delta_{0}(p, n)=\frac{1}{\sqrt{n}} \sum_{r \in K} c_{r} \sqrt{p^{r}\left(1-p^{r}\right.}\right)$; it is sufficient to prove that for any $\varepsilon>0$, player I can guarantee $f(p)-\delta_{0}(p, n)-\varepsilon$ (since $\Gamma_{n}(p)$ is a finite game he has an optimal strategy and can thus also defend $\left.f(p)-\delta_{0}(p, n)\right)$. Look at the equivalent game to $\Gamma_{n}(p)$ described in theorem 3.1. For any $\varepsilon>0$ consider the following strategy of player I: As long as $u\left(p_{m}\right) \geq f\left(p_{m}\right)$, play optimally in $\Delta\left(p_{m}\right)$ at stage-m. If $u\left(p_{m}\right)<f\left(p_{m}\right)$ then consider the $p_{m, h}$ and $\lambda_{h}$ of lemma 6 for $\frac{\varepsilon}{n}$.

Now, make the lottery described in lemma 6 , after which the a posteriori probability distribution is one of the $\left\{p_{m, h}\right\}$, say $p_{m, h}$; then play optimally in $\Delta\left(p_{m, h}\right)$ at stage- $m$. By this strategy, the expected payoff at stage- $m$ is (by lemma 3 ) at least:

- if $u\left(p_{m}\right) \geq f\left(p_{m}\right): u\left(p_{m}\right)-\sum_{r} c_{r} E\left(\left|p_{m+1}(r)-p_{m}(r)\right|\right) \geq f\left(p_{m}\right)-\sum_{r} c_{r} E\left|p_{m+1}^{r}-p_{m}^{r}\right|$

- if $u\left(p_{m}\right)<f\left(p_{m}\right)$, and $h$ is chosen (by argument similar to that used in proposition 4.2.):

$$
u\left(p_{m, h}\right)-\sum_{r} c_{r} E\left(\mid p_{m+1}(r)-p_{m}(r \mid h) \| h\right) \geq w\left(p_{m, h}\right)-\sum_{r} c_{r} E\left(\left|p_{m+1}(r)-p_{m}(r \mid h)\right| i h\right)
$$

where $w(p)=\min \{u(p), f(p)\}$.

Denote by $\tilde{p}_{m}(r)$ the random variable $p_{m}(r \mid h)$. If we take expectations with respect to $K$, we get:

$$
\sum \lambda_{h} w\left(p_{m, h}\right)-\sum_{r} c_{r} E\left(\left|p_{m+1}(r)-\tilde{p}_{m}(r)\right|\right) \geq f\left(p_{m}\right)-\frac{\varepsilon}{n}-\sum_{r} c_{r} E\left|p_{m+1}(r)-\tilde{p}_{m}(r)\right| .
$$

Let us define in the first case $\tilde{p}_{m}(r)=p_{m}(r)$. Then the sequence

$$
\left(p_{1}(r), \tilde{p}_{1}(r), \ldots, p_{m}(r), \tilde{p}_{m}(r), p_{m+1}(r), \ldots, p_{n}(r)\right)
$$


is also a martingale, and in any case, the expected payoff at stage- $m$ is at least

$$
f\left(p_{m}\right)-\frac{\varepsilon}{n}-\sum_{r} c_{r} E\left(\left|p_{m+1}(r)-\tilde{p}_{m}(r)\right|\right)
$$

Let us now prove that $f\left(p_{m}\right)+\frac{m-1}{n} \varepsilon, f\left(\tilde{p}_{m}\right)+\frac{m}{n} \varepsilon, f\left(p_{m+1}\right)+\frac{m}{n} \varepsilon, \ldots$ is a submartingale. For the steps from $f\left(p_{m}\right)+\frac{m-1}{n} \varepsilon$ to $f\left(\tilde{p}_{m}\right)+\frac{m}{n} \varepsilon$, it is trivial in the first case, and in the second case it follows immediately from (lemma 6): $f\left(p_{m}\right)-\frac{\varepsilon}{n} \leq \sum_{h} \lambda_{h} f\left(p_{m, h}\right)$. For the steps from $f\left(\tilde{p}_{m}\right)+\frac{m}{n} \varepsilon$ to $f\left(p_{m+1}\right)+\frac{m}{n} \varepsilon$, it follows from the fact that, since player I plays NS at $\tilde{p}_{m}, p_{m+1} \in \Pi_{\text {II }}\left(\tilde{p}_{m}\right)$ and since $f$ is convex w.r.t. II.

So we have $E\left(f\left(p_{m}\right)\right) \geq f(p)-\frac{m-1}{n} \varepsilon$. Thus the expected payoff at stage- $m$ is at least $f(p)-\frac{m-1}{n} \varepsilon-\frac{\varepsilon}{n}-\sum_{r} c_{r} E\left(\left|p_{m+1}(r)-\tilde{p}_{m}(r)\right|\right)$.The expected payoff over the whole game is then (by lemma 4) at least $f(p)-\delta_{0}(p, n)-\frac{1}{n}\left(\sum_{m=1}^{n} \frac{m}{n}\right) \varepsilon \geq$ $f(p)-\delta_{0}(p, n)-\frac{\varepsilon}{2}$ which completes the proof of the proposition.

Corollary.

$$
\underline{v}(p)=\vec{v}(p)=\lim _{n \rightarrow \infty} v_{n}(p)
$$

Proof.

Follows from (4.7) (ii), propositions 4.2 and 4.3 .

Definition.

Let us define $v(p)=\lim _{n \rightarrow \infty} v_{n}(p)$.

Theorem 4.4.

Consider the inequalities:

$(\alpha) f(p) \geq$ Cav Vex $\max \{u(p), f(p)\}$

$(\beta) f(p) \leq \underset{\text { II }}{\text { I }} \underset{\text { I }}{\text { II }} \min \{u(p), f(p)\}$.

Then

(A) $v(p)$ is the smallest solution of $(\alpha)$ and the largest solution of $(\beta)$.

(B) In particular $v(p)$ is the only solution of the system $\{(\alpha),(\beta)\}$.

(C) $v(p)$ is the only solution of the system:
$\left(\alpha^{\prime}\right) g(p)=\operatorname{Vex} \max \{u(p), g(p)\}$
$\left(\beta^{\prime}\right) g(p)=\underset{\mathrm{I}}{\mathrm{Cav}} \min \{u(p), g(p)\}$. 
Proof.

(A) and (B) follow immediately from propositions (4.1), (4.2), and 4.3 (with its dual). As for $(\mathrm{C})$, since $v(p)$ is convex w.r.t. II $v(p) \leq \underset{\mathrm{II}}{\operatorname{Vex}} \max \{u(p), v(p)\}$. Together with $(\mathrm{A})$, this implies that $v(p)$ is a solution of $\left\{\left(\alpha^{\prime}\right),\left(\beta^{\prime}\right)\right\}$. Uniqueness follows then from (B).

Theorem 4.5. (The Error term)

$$
-\frac{1}{\sqrt{n}} \sum_{r \in K} c_{r} \sqrt{p_{r}\left(1-p_{r}\right)} \leq v_{n}(p)-v(p) \leq \frac{1}{\sqrt{n}} \sum_{r \in K} c_{r}^{\prime} \sqrt{p_{r}\left(1-p_{r}\right)}
$$

where the $c_{r}^{\prime}$ are defined for player II in an analogous way as the $c_{r}$ were defined for player I.

Proof.

Follows immediately from proposition 4.3 and theorem 4.4 .

\section{Remarks.}

1) In the case where there are only two games, with lack of information on one side, we can get a somewhat better bounds, if we are a little more careful in lemma 3 ; in fact, define

$$
C_{r}(p)=\max _{j}\left|a_{j}^{r}(p)\right|, C=\max _{p}\left(C_{1}(p)+C_{2}(p)\right)
$$

Then $v(p)-v_{n}(p) \leq \frac{C \sqrt{p(1-p)}}{\sqrt{n}}$, and similarly for a lower bound.

2) In ZAMIR [1969], p. 6, the following example is given of a case where there are only two games, with lack of information for player II :

$$
\begin{array}{r|r|r|}
\hline p & -1 \\
\hline-3 & 1 \\
\hline 2-p & -2 \\
\hline-2 & 2 \\
\hline
\end{array}
$$

In this case, $u(p)=v(p)=0, v_{n}(p) \geq 0$, and we can show that, for $p=\frac{1}{2}$, the error term is bigger than $75 \%$ of the upper bound given here. (For $n=1$, the error term is even actually equal to the upper bound). The method is similar to that of ZAMIR [1969].

\section{Existence and Uniqueness of the Solution of Equations (2.2)}

In this section we will study somewhat more carefully the equations (2.2). We want to show that, for any continuous function $u$ on the simplex, there exists a continuous function $v=\varphi(u)$ on the simplex, such that theorem 4.4 remains 
valid. That is, we want to dispense in theorem 4.4 with the assumption that the function $u$ arises from some game.

Denote by $C$ the space of all continuous functions on the simplex; by $U$, the subset of $C$ consisting of those functions which are the " $u$-function" of some two-person zero-sum game with incomplete information. Denote by $\varphi$ the mapping $\varphi: U \rightarrow C, \varphi: u \rightarrow \varphi(u)=v$. In the whole of this paragraph, $C$ is endowed with the topology of uniform convergence.

\section{Proposition 5.1.}

a) $U$ is a vector lattice ${ }^{1}$ ) which contains all the affine functions.

b) In particular, $U$ is dense in $C$.

\section{Proof.}

a) 1) $U$ contains the affine functions.

Let $u=a_{0}+\sum_{1}^{k} a_{i} p_{i}=\sum_{1}^{k}\left(a_{i}+a_{0}\right) p_{i}$. Then $u$ is obviously the " $u$-function" of the game where $A^{i}=\left(a_{i}+a_{0}\right)$.

2) $u \in U \Rightarrow-u \in U$.

If $u$ arises from the game with matrices $\left(A^{1}, \ldots, A^{k}\right)$, then $-u$ arises from the game with matrices $\left[-\left(A^{1}\right)^{\prime},-\left(A^{2}\right)^{\prime}, \ldots,-\left(A^{k}\right)^{\prime}\right]$, where the prime denotes transposition.

3) $u \in U, \lambda \geq 0 \Rightarrow \lambda u \in U$.

If $u$ arises from $\left(A^{1}, \ldots, A^{k}\right)$, then $\lambda u$ arises from $\left(\lambda A^{1}, \ldots, \lambda A^{k}\right)$.

4) $u_{1} \in U, u_{2} \in U \Rightarrow u_{1}+u_{2} \in U$.

Let $u_{i}$ arise from $\left(A^{1, i}, \ldots, A^{k, i}\right)$, with pure strategy sets $M_{i}=\left\{1, \ldots, m_{i}\right\}$ for player I and $L_{i}=\left\{1, \ldots, l_{i}\right\}$ for player II. Then $u_{1}+u_{2}$ arises from $\left(B^{1}, \ldots, B^{k}\right)$, where $B^{h}(1 \leq h \leq k)$ is an $m_{1} m_{2} \times l_{1} l_{2}$ matrix, with elements

$$
b_{\left(i_{1}, i_{2}\right),\left(j_{1}, j_{2}\right)}^{h}=a_{i_{1}, j_{1}}^{h, 1}+a_{i_{2}, j_{2}}^{h, 2} \quad\left(\left(i_{1}, i_{2}\right) \in M_{1} \times M_{2},\left(j_{1}, j_{2}\right) \in L_{1} \times L_{2}\right) .
$$

5) $u \in U \Rightarrow u^{+} \in U$.

It is sufficient to add a last row of zeroes to each of the matrices $A^{i}-(u$ arises from $\left(A^{1}, \ldots, A^{k}\right)$ - in order to get the matrices $\left(A_{0}^{1}, \ldots, A_{0}^{k}\right)$ from which $u^{+}$arises.

These five points achieve the proof of (a).

(b) is a consequence from (a) and from the STONE-WeIERSTRASS theorem for lattices ${ }^{2}$ ), since the affine functions are clearly separating.

Proposition 5.2.

$\varphi: U \rightarrow C$ has a unique monotone extension $\varphi: C \rightarrow C-$ or: a unique continuous extension $\varphi: C \rightarrow C$; this extension is monotone and of norm 1 $(\|\varphi(f)-\varphi(g)\| \leq\|f-g\|)$.

\footnotetext{
1) A vector space which is a lattice.

2) See for instance "Real and Abstract Analysis" by Hewitt and STromberg, Springer-Verlag, N. Y., Inc., 1965. Theorem (7.29), p. 94.
} 


\section{Proof.}

$\varphi: U \rightarrow C$ is monotone and of norm 1. Indeed, monotonicity follows immediately from theorem 4.4 (A). The fact that $\varphi$ is of norm 1 follows from monotonicity and from the fact that, if $\varepsilon$ is any constant, $\varphi(u+\varepsilon)=\varphi(u)+\varepsilon-$ which is also a trivial consequence of theorem 4.4. Therefore, the fact that $U$ is dense in $C$ (proposition 5.1) gives immediately the proposition.

\section{Theorem 5.3.}

Theorem 4.4 remains true for any $u \in C$, with $v$ replaced by $\varphi(u)$.

\section{Proof.}

Since the operators Cav, Vex, max, and min used in the several equations of theorem 4.4 are continuous with respect to the supremum norm, and since $\varphi$ is continuous with respect to that norm, we have immediately that

$\left(1^{\prime}\right) \varphi(u)(p)=\underset{\text { II }}{\operatorname{Vex} \max }\{u, \varphi(u)\}$

$\left(2^{\prime}\right) \varphi(u)(p)=\underset{\mathrm{I}}{\mathrm{Cav}} \min \{u, \varphi(u)\}$.

In particular, $\varphi(u)$ is a solution of

(1) $\varphi(u)(p) \geq \underset{\text { I II }}{\operatorname{Cav}} \operatorname{Vex} \max \{u, \varphi(u)\}$.

If we prove it is the smallest solution of (1), then we can achieve the proof of theorem 5.3 in the same way as we proved theorem 4.4 .

So, let $f$ be any solution of $(1)$, and let $\left(u_{n}\right)$ be an increasing sequence in $U$ converging uniformly to $u$ (this exists by proposition 5.1). Then $f$ is a fortiori a solution of $f(p) \geq$ Cav Vex $\max \left\{u_{n}, f\right\}$, and so, by theorem $4.4,(\mathrm{~A}), f(p) \geq \varphi\left(u_{n}\right)(p)$. But, by the continuity of $\varphi, \varphi\left(u_{n}\right) \rightarrow \varphi(u)$, and so $f \geq \varphi(u)$. This completes the proof of theorem 5.3.

Theorem 5.4. (An approximation procedure for $\varphi(u)$ )).

Define

$$
\underline{v}_{0}=-\infty, \bar{v}_{0}=+\infty, \underline{v}_{n+1}=\underset{\text { I }}{\operatorname{Cav}} \operatorname{Vex} \max \left\{u, \underline{v}_{n}\right\}, \bar{v}_{n+1}=\underset{\text { II }}{\operatorname{Vex}} \operatorname{Cav} \min \left\{u, \bar{v}_{n}\right\} .
$$

Then $\underline{v}_{n} \leq \underline{v}_{n+1} \leq \cdots, \bar{v}_{n} \geq \bar{v}_{n+1} \geq \cdots$, and both $\left(\underline{v}_{n}\right)$ and $\left(\bar{v}_{n}\right)$ converge uniformly to $\varphi(u)$.

\section{Proof.}

Since $u$ is continuous on a compact set, it is uniformly continuous, and it is easy to check that the operators min, max, Vex and Cav preserve the modulus of uniform continuity. Therefore, both sequences $\left(\underline{v}_{n}\right)_{n=1, \ldots}$ and $\left(\bar{v}_{n}\right)_{n=1, \ldots}$ are equicontinuous, and obviously bounded: so they are compact in the uniform topology. 
Let us prove $\underline{v}_{n} \leq \underline{v}_{n+1}$. It is obviously true for $n=0$. If $\underline{v}_{n-1} \leq \underline{v}_{n}$, then $\underline{v}_{n}=\underset{\text { I }}{\operatorname{Cav}} \operatorname{Vex} \max \left\{u, \underline{v}_{n-1}\right\} \leq \underset{\text { II }}{\operatorname{Cav}} \operatorname{Vex} \max \left\{u, \underline{v}_{n}\right\}=\underline{v}_{n+1}$.

Let us prove $\underline{v}_{n} \leq \varphi(u)$. It is obviously true for $n=0$. If $\underline{v}_{n-1} \leq \varphi(u)$, then $\underline{v}_{n}=$ Cac Vex $\max \left\{u, \underline{v}_{n-1}\right\} \leq$ Cav Vex $\max \{u, \varphi(u)\} \leq \varphi(u)$ (by theorem 5.3).

Let $v=\lim _{n \rightarrow \infty} \underline{v}_{n}$ : this limit is uniform by the compactness of the sequence. Then $\underline{v}=\underset{I}{\operatorname{Cav}} \operatorname{Vex} \max \{u, \underline{v}\}$, and $\underline{v} \leq \varphi(u)$. Theorem 5.3 implies, therefore, that $\underline{v}=\varphi(u) ; \underline{v}_{n}$ converges uniformly to $\varphi(u)$. The same argument applies to the sequence $\bar{v}_{n}$.

Corollary 5.5 .

If $u$ is a continuous function on the simplex, then both Cav Vex $u$ and Vex Cav $u$ are convex w.r.t. II and concave w.r.t. I.

\section{Proof.}

Define $v=\operatorname{Vex} u$. Then $\varphi(v)=\operatorname{Vex} \max \{v, \varphi(v)\}$. Since both $v$ and $\varphi(v)$ are convex w.r.t. II, $\max \{v, \varphi(v)\}$ is also; therefore, $\varphi(v)=\max \{v, \varphi(v)\}: \varphi(v) \geq v$. But $\varphi(v)=\operatorname{Cav} \min \{v, \varphi(v)\}=\operatorname{Cav} v=\operatorname{Cav} \operatorname{Vex} u$. Since $\varphi(v)$ is convex w.r.t. II, it follows that Cav Vex $u$ is also. From this follows the corollary.

\section{Remark 1 .}

On alternative set-ups for this paper.

The results of this paper can be obtained by several other approaches. Let us sketch here some of them.

a) (i) If player I can guarantee $f(p)$ (up to an $\varepsilon$ ), then he can also guarantee Cav Vex $\max \{u(p), f(p)\}$. The proof of this is completely similar to that of ProI II position 4.1, using also Proposition 4.2. A dual statement is valid for player II.

(ii) Define a sequence $\underline{v}_{n}$ and a sequence $\bar{v}_{n}$ as in theorem 5.4. Then applying the above property inductively it follows that player I can guarantee each of the $v_{n}$ and therefore he can also guarantee $\underline{v}=\lim \underline{v}_{n}$. Similarly, player II can guarantee

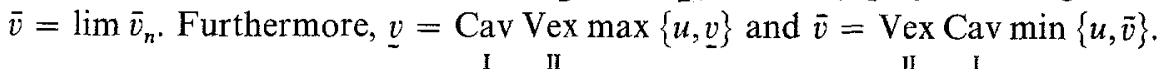

(iii) From these two equations and the fact that $\underline{v} \leq \bar{v}$ one derives that $\underline{v}=\bar{v}$ (as in MERTENS and ZAMIR, theorem 4.2).

b) (i) The first point is as in (a).

(ii) Define $\underline{v}=\lim \inf v_{n}$ and $\bar{v}=\lim \sup v_{n}$. Player I can guarantee $\underline{v}$ and player II can guarantee $\bar{v}$. So replace $f$ in the first point by $\underline{v}$ (and by $\bar{v}$ in the dual statement for player II).

(iii) The third point is again identical to the third point of (a).

This is the way used by MERTENS and ZAMIR. The first two points are essentially equivalent to Proposition 4.1 and 4.2. A third approach would be: 
(i) Prove directly corollary 5.5.

(ii) Using this and theorem 5.4, prove theorem 5.3 [through MERTENS and ZAMIR, theorem 4.2.].

(iii) Prove Proposition 4.3 and deduce the required result by applying it to both players.

\section{Remark 2.}

It is quite easy to see that several other limiting procedures such as discounting, lead to the same limit with a similar error term. In fact, now that we have the existence and uniqueness of $v=\varphi(u)$ for each continuous $u$ (theorem 5.3), Proposition 4.3 applies immediately to each of the other cases, with a slight variation in the computation of the error term. For instance:

a) Discounting. For $0<\beta<1$ let $H_{\beta}(p)=E\left(\sum_{0}^{\infty} \beta(1-\beta)^{n} H_{n+1}\right)$, where $H_{n+1}$ is the payoff at stage $n+1$. Then $H_{\beta}(p)$ is the payoff function of what we might call the discounted game.

One can think of this game as if there was a stopping time $T$, with a geometric distribution such that at each stage $n$ there is a probability $\beta$ of stopping at that stage, given that no stop occured before. $T$ is independent of the game and is not told to the players. Then $H_{\beta}(p)$ can be looked upon in either of the two following ways: either the players get as payoff the payoff they received at stage $T$ exactly (not the accumulated payoff) - or the players get as payoff their accumulated payoff up to stage $T$, divided by $\frac{1}{\beta}$, the expected number of stages in the game.

Let $v_{\beta}(p)$ be the value of this game. Then $v_{\beta}(p) \geq v(p)-\sum_{r} c_{r} \sqrt{p^{r}\left(1-p^{r}\right)} \frac{1}{\sqrt{\frac{1}{\beta}}}$. Thus as $\beta \rightarrow 0$ we get (using also the dual inequality) $v_{\beta}(p) \rightarrow v(p)$ with an error term of the order of magnitude $1 / \sqrt{1 / \beta}$ which is the analogue of $1 / \sqrt{n}$.

b) A Variant of (a) might be to give the players as payoff their accumulated payoff up to time $T$, divided by the actual number of stages in the game - this means $H_{\beta}(p)=E\left(\sum_{0}^{\infty} \beta(1-\beta)^{n} \sum_{1}^{n+1} \frac{H_{i}}{n+1}\right)$ - then one gets:

$$
\begin{aligned}
v_{\beta}(p) & \geq v(p)-\sum_{r} c_{r} \sqrt{p^{r}\left(1-p^{r}\right)} \frac{\beta \sqrt{\pi}}{(1-\beta) \sqrt{\ln \frac{1}{1-\beta}}} \\
& \geq v(p)-\sum_{r} c_{r} \sqrt{p^{r}\left(1-p^{r}\right)} \frac{\sqrt{\pi}}{1-\beta} \frac{1}{\sqrt{\frac{1}{\beta}}}
\end{aligned}
$$

With the same interpretation for $1 / \beta$, the analogy is again striking $(\sqrt{\pi / 1}-\beta$ is essentially constant when $\beta \rightarrow 0$ ).

This shows really that our results remain essentially unchanged for any reasonable concept of an "often repeated game". 


\section{Examples}

In this last section let us find $v(p)$ for some examples. The examples are what we called product games. In these games our representation leads to the usual one used by Aumann and MASCHLER [1967] and STEARns.

By rescaling probabilities and payoffs in a compensating manner, it can be shown that any game of the type we considered in this paper is strategically equivalent to such a product game and even one with uniform probabilities. Indeed, if the original game were expressed in our usual notation, the matrices $B^{i j}$ for the equivalent game would be given by the equations:

$$
B^{i j}=\mu \cdot v \cdot \sum_{k \in K_{1}^{i} \cap K_{J}^{\mathrm{II}}} p_{k} A_{k}
$$

where an empty sum is zero.

This shows that our restriction to examples of the product type is not a severe restriction. Indeed, this transformation permits to reduce each game, for fixed $p$, to a product game, and thus to compute its solution - for each fixed $p$. But it makes it not possible to see the dependence of the solution on $p$. We owe this remark to the referee. $p$ will denote from now on the probability distribution assigned by player II to the types of player I and $q$ is the probability distribution of player I on the types of player II. The game will be denoted by $\Gamma_{n}(p, q)$, its value by $v_{n}(p, q), \lim _{n \rightarrow \infty} v_{n}(p, q)=v(p, q)$ and $u(p, q)$ will be the value of $\Delta(p, q)$, the game in which both players play NS.

The equations (2.2) which determine $v(p, q)$ are now:

$$
\begin{aligned}
\text { i) } v(p, q) & =\underset{q}{\operatorname{Vex} \max }\{u(p, q), v(p, q)\} \\
\text { ii) } v(p, q) & =\underset{p}{\operatorname{Cav} \min }\{u(p, q), v(p, q)\} .
\end{aligned}
$$

In the following examples, there are two types of each player, so $p$ and $q$ are one dimensional and the functions can be described on the unit square. Even in this simple case, we do not have in general an explicit solution of equations (6.1). However, it turns out that the most useful result is lemma 6 of $\S 4$ which for this case says:

i) $u(p, q)<v(p, q) \Rightarrow v(p, q)$ is linear in the $p$ direction.

ii) $u(p, q)>v(p, q) \Rightarrow v(p, q)$ is linear in the $q$ direction.

So we can describe $v(p, q)$ by giving only the locations $(p, q)$ on the unit square on which $v(p, q)=u(p, q)$.

We will write shortly $p^{\prime}$ for $1-p$ and $q^{\prime}$ for $1-q$.

\section{Example 1}

The first example is that considered in AUMANN and MASCHLER [1967], in which $\operatorname{Cav} \operatorname{Vex} u(p, q) \neq \operatorname{Vex} \operatorname{Cav} u(p, q)$ and hence $v_{\infty}(p, q)$ does not exist. The matrices $p \quad q \quad q \quad p$ are as follows: 


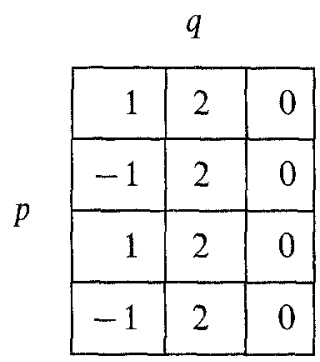

\begin{tabular}{|r|r|r|}
\multicolumn{1}{c}{$q^{\prime}$} \\
\hline-1 & 0 & 2 \\
\hline 1 & 0 & 2 \\
\hline 1 & 0 & 2 \\
\hline-1 & 0 & 2 \\
\hline
\end{tabular}

\begin{tabular}{|r|r|r|}
\hline 1 & 0 & 2 \\
\hline-1 & 0 & 2 \\
\hline-1 & 0 & 2 \\
\hline 1 & 0 & 2 \\
\hline
\end{tabular}

\begin{tabular}{|r|r|r|}
\hline-1 & 2 & 0 \\
\hline 1 & 2 & 0 \\
\hline-1 & 2 & 0 \\
\hline 1 & 2 & 0 \\
\hline
\end{tabular}

The functions $u(p, q)$ and $v(p, q)$ are given in the following diagrams.

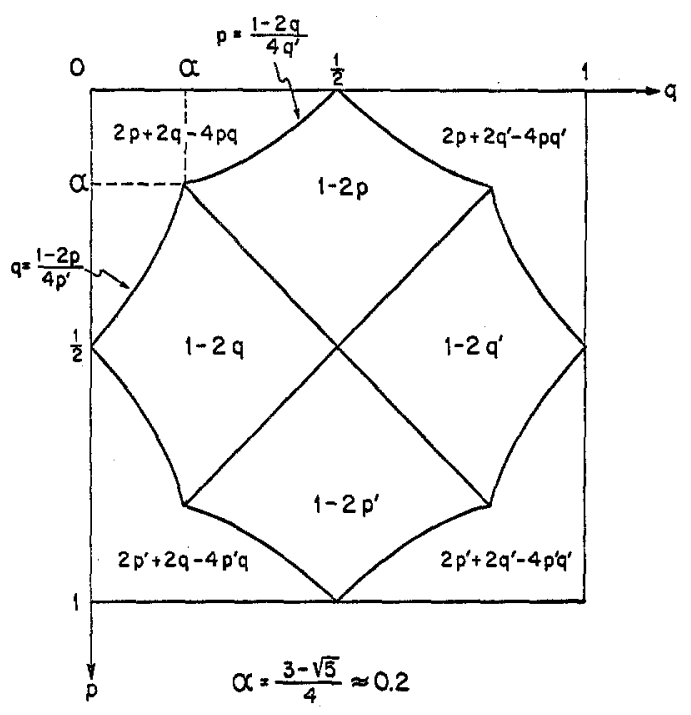

Fig. 1: $u(p, q)$ of example 1

It turns out that $v(p, q)=\lim v_{n}(p, q)=\operatorname{Cav} \operatorname{Vex} v(p, q)$ as shown in Figure 2. ${ }_{n \rightarrow \infty} \quad p \quad q$

The thick lines are the lines on which $v(p, q)=u(p, q)$. On the regions defined by these lines $v(p, q)$ is linear in the directions described by the arrows (according to whether $u(p, q)>v(p, q)$ or $u(p, q)<v(p, q)$ ). 


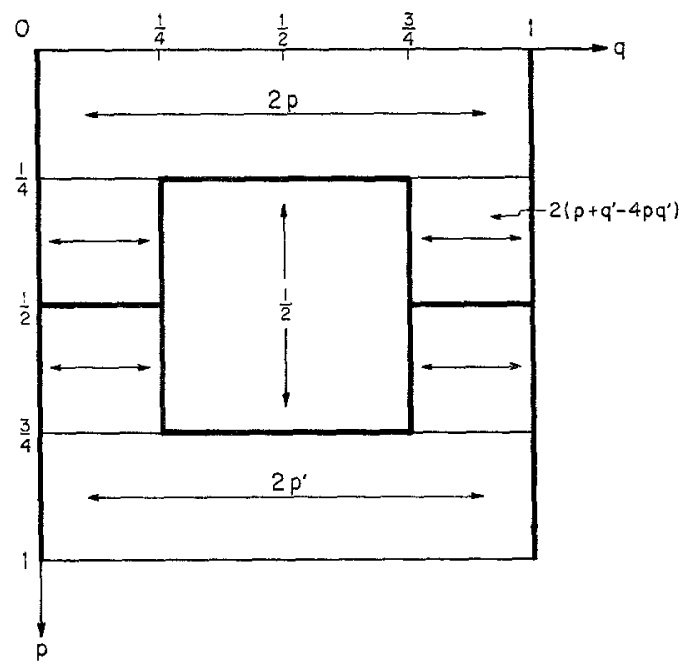

Fig. 2: $\underset{p}{\operatorname{Cav}} \underset{q}{\operatorname{Vex}} u(p, q)=v(p, q)$ of example 1

So this example happens to be somehow a special case, namely, $\lim v_{n}$ coincides with of the two bounds Cav Vex $u$ and Vex Cav $u$. The second example does not have this property.

Example 2.

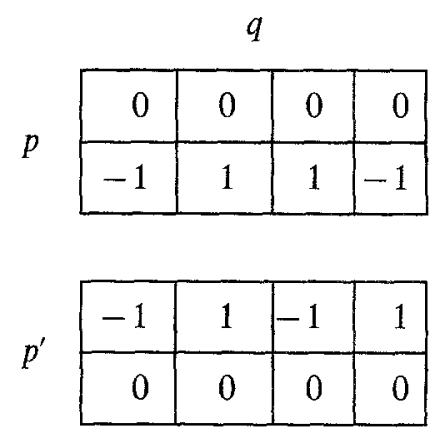

$q^{\prime}$
\begin{tabular}{|r|r|r|r|}
\hline 1 & -1 & 1 & -1 \\
\hline 0 & 0 & 0 & 0 \\
\hline 0 & 0 & 0 & 0 \\
\hline 1 & -1 & -1 & 1 \\
\hline
\end{tabular}

$$
\text { Here } \Delta(p, q)=\begin{array}{|l|l|l|l|}
\hline p-q & q-p & p-q & q-p \\
\hline q^{\prime}-p & p-q^{\prime} & p-q^{\prime} & q^{\prime}-p \\
\hline
\end{array}
$$

The functions $u(p, q), \operatorname{Cav} \operatorname{Vex} u(p, q)$ and $\operatorname{Vex} \operatorname{Cav} u(p, q)$ are given in Figures 3 to 5 . Notice that all the functions under consideration are symmetric with respect to $p=\frac{1}{2}$ and $q=\frac{1}{2}$. 


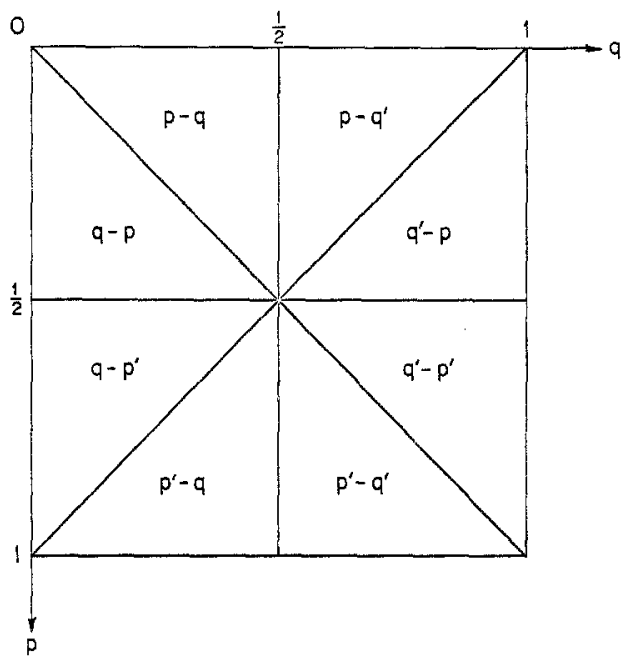

Fig. 3: $u(p, q)$ of example 2

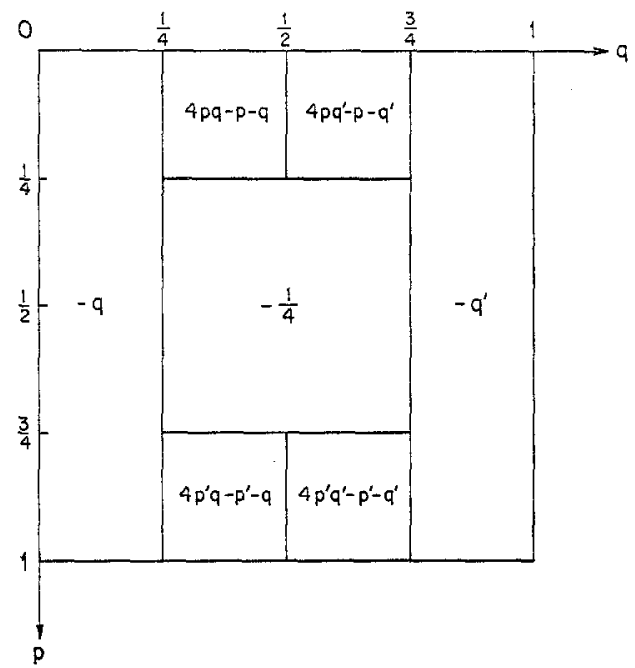

Fig. 4: $\underset{p}{\operatorname{Cav} \operatorname{Vex}} u(p, q)$ of example 2

To find $v(p, q)$ we proceed through the following steps:

1. On the segment $\left(0, \frac{1}{2}\right)-\left(\frac{1}{4}, \frac{1}{2}\right)(A-B$ on the fig. of Vex Cav) Cav Vex $=$ Vex $\mathrm{Cav}=u$, so this must also be the value of $v$ on this segment.

2. On $(0,0)$ (and so on the four extreme points) we have Cav Vex $=\mathrm{Vex} \mathrm{Cav}=u$, so again this must also be the value of $v$ there. 


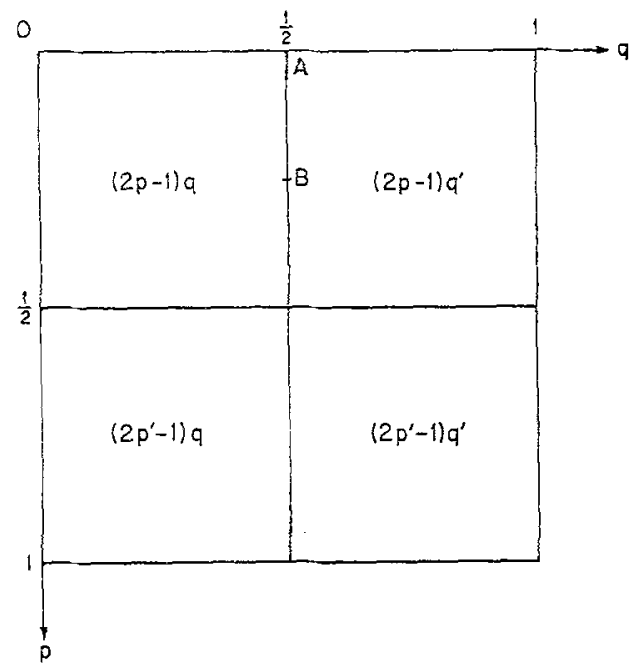

Fig. 5: Vex Cav $u(p, q)$ of example 2

3. On the diagonals we have $u>$ Vex Cav (except in the center, and, of course, on the extreme points), so certainly $u>v$ there.

4. On the sides $(0,0)-(1,0)$, (except the end points) we have $u<$ Cav Vex and so certainly $u<v$. We conclude that the line on which $v=u$ (in the first quarter) starts at $(0,0)$ and lies between the diagonal and the side $(0,0)-\left(\frac{1}{2}, 0\right)$.

5 . The second line on which $v=u$ which contains $\left(0, \frac{1}{2}\right)-\left(\frac{1}{4}, \frac{1}{2}\right)$ (see 1) must continue beyond $\left(\frac{1}{4}, \frac{1}{2}\right)$ but not as far as $\left(\frac{1}{2}, \frac{1}{2}\right)$ otherwise convexity in the $q$ direction is violated. Let the endpoint of this segment be $\left(\xi, \frac{1}{2}\right),\left(\frac{1}{4}<\xi<\frac{1}{2}\right)$.

6. At any point on which $v=u, v$ is differentiable in both variables jointly if $u$ itself is. (This last property is general, provided the line is not parallel to the axes).

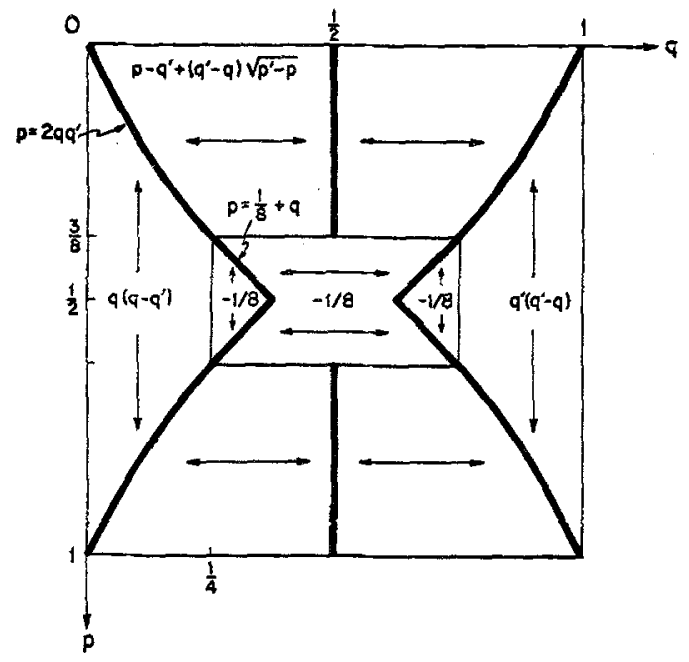

Fig. 6: $v(p, q)$ of example 2 
After the above considerations, we let $p=f(q)$ be the line between the diagonal and the side on which $v=u$. Then we write $v(p, q)$ in terms of $f$ by linearity considerations. 6. gives, then, a differential equation for $f(q)$. The solution of this equation happens to be $p=2 q q^{\prime}$. From this we find (by convexity arguments) the parameter $\xi$ (which happened to be $\frac{3}{8}$ ). Finally, we write again the differential equation from $p=\frac{3}{8}$ to $p=\frac{1}{2}$ and find $p=\frac{1}{8}+q$ for $\frac{1}{4} \leq q \leq \frac{3}{8}$. The resulting $v(p, q)$ is given in Figure 6.

As in the previous example, thick lines are the locations on which $v=u$ and the arrows denote the directions of the linearity of $v$.

One can check that the $v(p, q)$ we get is indeed a solution of (6.1). This is done easily and so the problem is solved. Notice that in this example $v(p, q)=\lim v_{n}(p, q)$ is almost everywhere different from Cav Vex $u$ and from Vex Cav $u$.

Example 3.

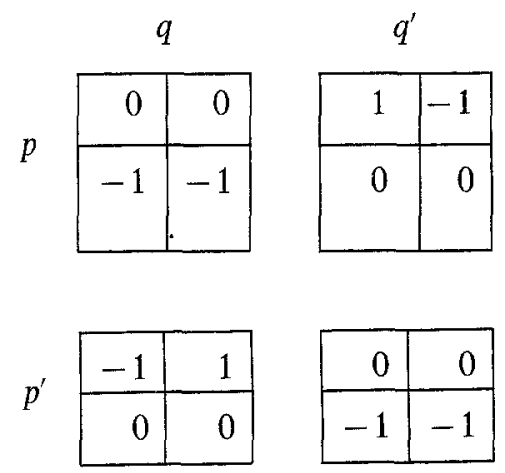

$$
\Delta(p, q)=\begin{array}{|c|c|}
\hline p-q & q-p \\
\hline-p q-p^{\prime} q^{\prime} & -p q-p^{\prime} q^{\prime} \\
\hline
\end{array}
$$

The function $u(p, q)$ is given in Figure 7 .

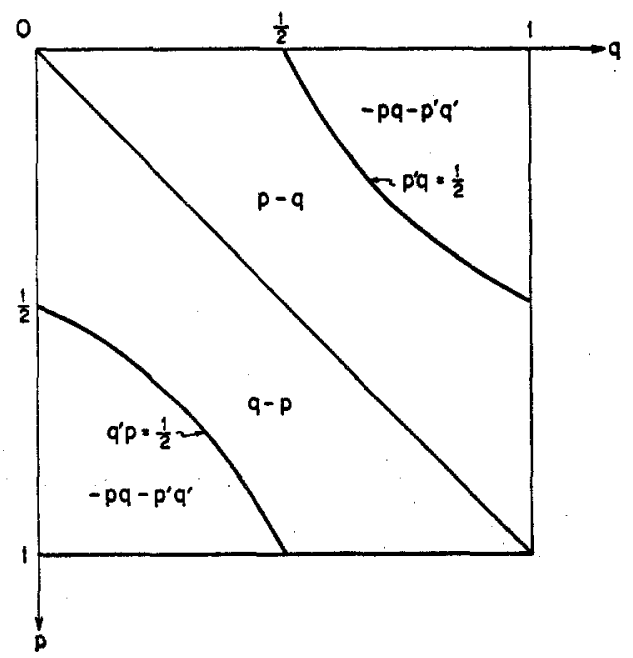

Fig. $7: u(p, q)$ of example 3 
Cav Vex $u$ and Vex Cav $u$ are obtained by quite tedious calculations and since they are not very important here, we do not give them. (They can be found in MERTENS and ZAMIR.) We will just remark that Cav Vex $u \neq$ Vex Cav $u$ almost everywhere.

The solution of the equations for $v(p, q)$ is made by considerations similar to those made in the previous example. The problem is reduced to one differential equation of the line on which $v=u$. (See Figure 8.)

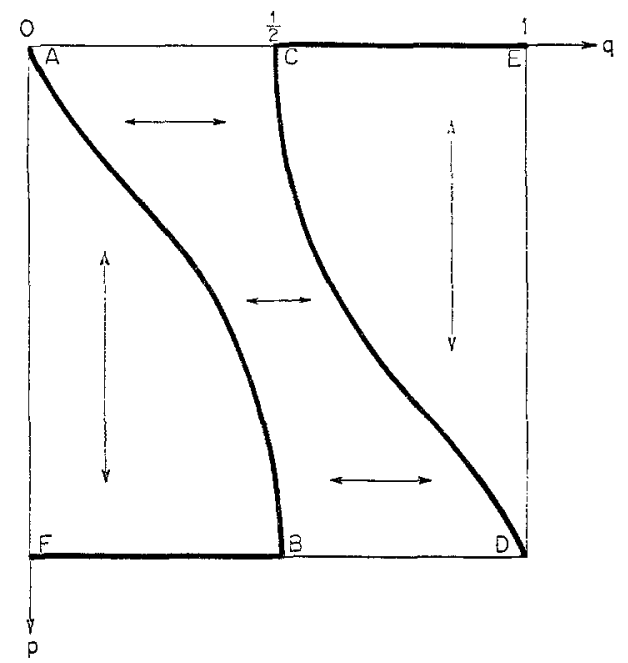

Fig. 8: $v(p, q)$ of example 3

Let $q=f(p)$ be the equation of the line $A B$ of Figure 8; then $q^{\prime}=f\left(p^{\prime}\right)$ is the equation of $C D$. These two lines and the two segments $C E$ and $F B$ are the lines on which $v(p, q)=u(p, q)$. The values of $v$ on the whole square are obtained by linearity in the directions of the arrows.

It can be shown by inspection of the figures that $f(p) \leq \frac{1}{2}, f(1)=\frac{1}{2}$. So $f(p)$ is the solution of the following equation:

$$
f^{\prime}(p)=\frac{(1-2 f(p))(1-f(p)-f(1-p))}{2(1-p)(1-p-f(1-p))}
$$

with $0 \leq p \leq 1, f(0)=0, f(1)=\frac{1}{2}$.

This equation we could not solve explicitly. Of course, it can be solved numerically, From the equation we could deduce that $f(p)$ is a transcendental function since all the derivatives at $p=1$ are 0 and the same for the derivatives at $p=0$, except the first, which is $\frac{1}{2}$.

The above equation can be transformed to any one of the two following systems of two ordinary differential equations by the substitutions: 


$$
\begin{gathered}
p=\frac{t+1}{2}, \quad \varphi(t)=\tilde{\varphi}\left(\frac{t+1}{2}\right), \quad \tilde{\varphi}(p)=1-2 f(1-p), \\
\psi(t)=\tilde{\psi}\left(\frac{t+1}{2}\right), \quad \tilde{\psi}(p)=1-2 f(p)
\end{gathered}
$$

A)

$$
\begin{aligned}
& \psi^{\prime}(t)=\psi(t) \frac{\psi(t)+\varphi(t)}{(1-t)(t-\varphi(t))} \\
& \varphi^{\prime}(t)=\varphi(t) \frac{\psi(t)+\varphi(t)}{(1+t)(t+\psi(t))}
\end{aligned}
$$

$$
\begin{aligned}
& -1 \leq t \leq 1 \\
& \psi(1)=0 ; \quad \varphi(1)=1 \\
& \varphi^{(n)}(1)=0 \quad \text { for } \quad n \geq 2 \\
& \psi^{\prime}(1)=\frac{1}{2} \\
& \varphi^{(n)}(1)=0 \\
& \psi(-t)=\varphi(t)
\end{aligned}
$$

B) $\frac{d \log \psi}{d \log \varphi}=\frac{1+t}{1-t} \frac{t+\psi}{t-\varphi}$

$$
\frac{d \log (1-t)}{d \log \psi}+\frac{d \log (1+t)}{d \log \varphi}=1 .
$$

\section{Acknowledgements}

J. F. Mertens is indebted to R. J. Aumann, who spent hours introducing him to this subject, in a long series of conversations, in the spring and summer, 1969. He wishes, here, to express all his thanks.

\section{References}

AumanN, R. J., and M. Maschler: Repeated Games with Incomplete Information. A survey of recent results. Report to the U. S. Arms Control and Disarmament Agency, Washington, D. C. Final report on contract ACDA/ST-116, prepared by Mathematica, Princeton, N. J., September, 1967, Chapter III, pp. $287-403$.

- : Repeated Games with Incomplete Information. The Zero-Sum Extensive Case. Report to the U. S. Arms Control and Disarmament Agency, Washington, D. C. Final report on contract ACDA/ST143, prepared by Mathematica, Princeton, N. J., November, 1968. Chapter II, pp. $25-108$.

HARSANYI, J. C.: Games with Incomplete Information Played by 'Bayesian' Players. Parts I, II, III. Management Science, Vol. 14, $\mathrm{n}^{\circ}: 3,5,7$.

Mertens, J.F., and S.ZAmir: The Value of Two-Person Zero-Sum Repeated Games with Lack of Information on Both Sides. Research Program in Game Theory and Mathematical Economics, Research Memorandum No.61, July, 1970, Institute of Mathematics, The Hebrew University of Jerusalem, Jerusalem, Israel.

StEARns, R. E.: A Formal Information Concept for Games with Incomplete Information. Report to the U. S. Arms Control and Disarmament Agency, Washington, D. C. Final report on contract ACDA/ST-116, prepared by Mathematica, Princeton, N. J., September, 1967. Chapter IV, pp. $405-433$.

ZaMIR, S.: On the Relation Between the Values of Finitely and Infinitely Repeated Games of Incomplete Information. Research Program in Game Theory and Mathematical Economics, Research Memorandum No. 43, January, 1969, Institute of Mathematics, The Hebrew University of Jerusalem, Jerusalem, Israel.

- : The Inequivalence Between to Two Approaches to Repeated Games with Incomplete Information. Research Program in Game Theory and Mathematical Economics, Research Memorandum No. 55, April, 1970, Institute of Mathematics, The Hebrew University of Jerusalem, Jerusalem, Israel. 